\title{
Massive vulval oedema in multiple pregnancies at Bugando Medical Centre: Case reports
}

\author{
ANTHONY N. MASSINDE*, NNHANDI NGWALIDA and BALTHAZAR GUMODOKA \\ Department of Obstetrics and Gynaecology, Bugando Medical Centre Box 1370 Mwanza, Tanzania
}

\begin{abstract}
Massive vulval oedema is not common during pregnancy, but when it develops, it often is associated with patient discomfort and management challenges. Two pregnant women presented to Bugando Medical Centre in Mwanza, Tanzania, with massive swelling of the vulva at 39 weeks and 32 weeks of gestation. Both women were found to have multiple gestations. Despite medical management, there was no resolution of the oedema. In both cases, vulval oedema subsided after being delivered by caesarean section. Major vulval oedema during pregnancy is a serious issue. If not treated appropriately, it can lead to major complications of increased blood loss and poor wound healing. Abdominally delivery is the preferred mode of delivery at our institution for massive vulval oedema.
\end{abstract}

Keywords: vulva, oedema, pregnancy, management, Tanzania

\section{Introduction}

Massive vulval oedema is not common during pregnancy (Deren et al., 2000; Saha, 2007), but when it develops, it is associated with patient discomfort and management challenges (Jakobi et al., 1995). Several cases of the massive vulval oedema has been described in the literature associated with multiple pregnancy, diabetes, hypoproteinemia, pre-eclampsia, tocolytic therapy, and vulvo-vaginitis (Morris et al., 1990; Jokobi et al., 1995; Trice et al., 1996; Deren et al., 2000; Reynolds, 2003; Saha, 2007). In this report we describe two cases of massive vulval oedema seen in two patients with multiple pregnancies.

\section{Case 1}

A 23 year old gravida two para one was admitted at gestational age of 39 weeks with complaint of lower limb swelling that was unrelated to headache, palpitations, or easy fatigability. On physical examination she had bilateral pre-tibial, sacral and labial oedema. She was afebrile and her blood pressure was 110/70 mmHg. Abdominal examination revealed a gravid uterus equivalent to 38 weeks, estimated foetal weight of four kilograms, and in cephalic presentation. Foetal heart rate was normal. Obstetric ultrasound showed diamniotic-dichorionic twins, both in cephalic presentation. Her haemoglobin was $9.44 \mathrm{~g} / \mathrm{dl}$ and urinalysis showed white blood cell count of more than 35/high power field and negative for protein. She was treated with nitrofurantoin as the urinalysis was suggestive of bacteriuria.

\footnotetext{
* Correspondence: Dr. Antony N. Massinde; E-mail: amassinde@yahoo.com
} 
While in the ward, vulval oedema increased, causing pain (vulvodynia) and passage of yellow-whitish discharge per vagina (Figure 1). Examination revealed massive oedema, and digital vaginal examination was difficult due to tenderness. Vulvovaginitis was suspected, and she was started on empiric cloxacillin, metronidazole, diclofenac and sitz bath. A vaginal swab taken for culture and microscopic smear were both negative.

Four days later, she developed labour pains, and an assessment of the cervical dilatation was impossible due to pain. Hence, decision was made to take her for caesarean section. Diamniotic-dichorionic twins, a male $2.4 \mathrm{~kg}$ and a female $2.3 \mathrm{~kg}$, both with Apgar scores of eight and nine at first and fifth minute, respectively, were delivered under general anaesthesia. Post operative progress was un-remarkable. She was given furosemide to reduce oedema. On post operative day 2 , swelling of the vulva was resolved. She was discharged six days later.

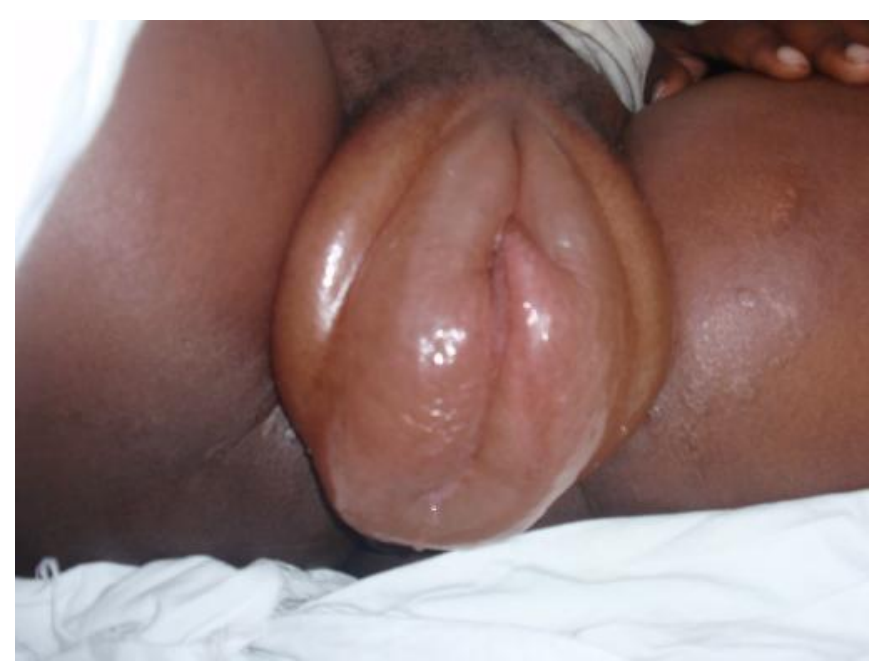

Figure 1: Vulval oedema in case: bilateral swollen labia minora and majora

\section{Case 2}

A 27 year old primigravida at gestational age of 32 weeks was admitted to our facility with a main complaint of swelling of external genitalia for two days. On examination she was found to be pale, afebrile, with lower limb oedema and a blood pressure of $150 / 80 \mathrm{mmHg}$. Abdominal examination revealed a gravid uterine fundus equivalent to 38 weeks, a foetus in cephalic presentation, and a normal foetal heart rate. Examination of the external genitalia revealed a swollen, tender vulva, with no obvious vaginal discharge (Figure 2).

Obstetric ultrasound revealed diamniotic-dichorionic twins with reduced amniotic fluid at 31 weeks of gestation age. Haemoglobin level was $6.8 \mathrm{~g} / \mathrm{dl}$. Urine dipstick revealed proteinuria of plus two and creatinine was within normal range. She was transfused one unit of blood, given iron supplements and started on oral cloxacillin as empirical treatment, diclofenac for pain relief and sitz bath with dettol solution 
She was schedule for a caesarean delivery at 34 weeks due to ongoing oedema and evolving pre-eclampsia. Diamniotic-dichorionic twins were delivered: first twin was female with birth-weight of $1.7 \mathrm{~kg}$ and Apgar score of two and seven at first and fifth minute respectively, second twin was a male with birth-weight of $2.07 \mathrm{~kg}$, and Apgar scores of one and six at first and fifth minute respectively. Vulval oedema resolved two days later after delivery.

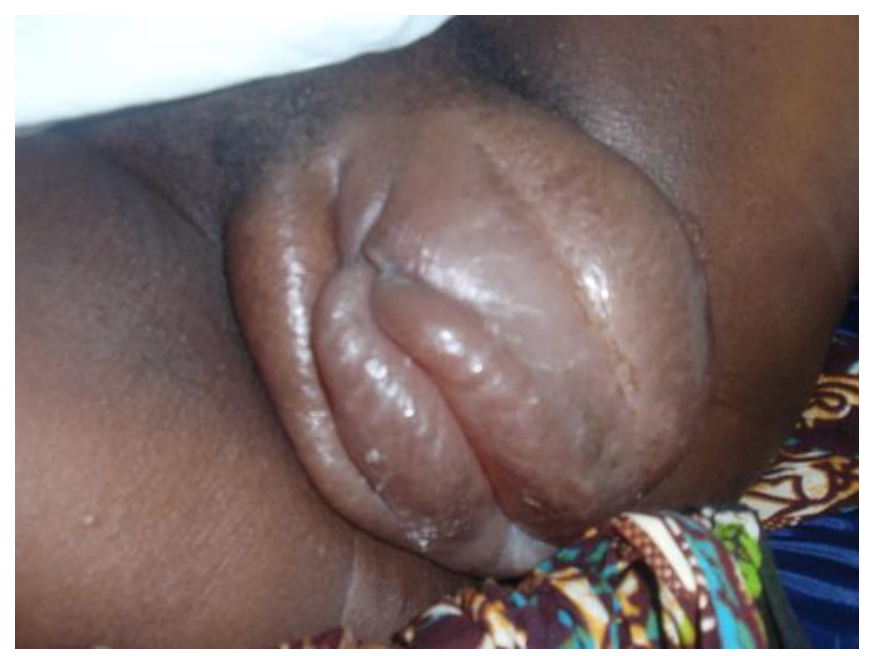

Figure 2: Vulval oedema in case 2

\section{Discussion}

Massive vulval oedema during pregnancy is usually benign. However there have been reports of maternal death associated with this condition after delivery (Morris et al., 1990; Deren et al., 2000; Reynolds, 2003; Saha, 2007). This condition should be taken seriously since it might be caused by some conditions such as pre-eclampsia or diabetes (Morris et al., 1990; Jokobi et al., 1995; Trice et al., 1996; Deren et al., 2000; Reynolds, 2003; Saha, 2007).

There is no standard way of managing this condition, as there were only few cases reported in the literature. It is important to find the cause, as management is influenced by the cause of oedema. Analgesics may be used when the pregnancy is remote from term or not about to go into labour and mechanical drainage have been tried in some occasion (Bracero \& Didomenico, 1991; Deren et al., 2000).

Several modes of delivery have been described in the literature. Puncturing the labial skin and allow vaginal birth has been tried successfully (Townsend, 1948). Reynolds (2003) reported using of epidural anaesthesia which allowed monitoring labour by assessment of cervical dilation. However, it is not known whether there is a risk of delayed healing of punctured skin/vulva tear or of super-infection in the presence of oedema. These concerns led us to opt for an abdominal delivery, as has been reported elsewhere (Bush, 1946; Jakobi et al., 1995)

In most cases, oedema of the vulva will resolve spontaneously after delivery (Jakobi et al., 1995) as in our case. In our cases the aetiology is most likely to be 
secondary to multiple gestations, with pre-eclampsia likely complicating the second case. In one of the cases, furosemide was administered to hasten resolution of oedema, which is important, as vulvodynia may prevent early ambulation and put the patient at risk of thrombo-embolism (Geerts et al., 2004). Although it helped with recovery in case 1 , it should not be taken as standard treatment.

Major vulval oedema during pregnancy is a serious problem. If not treated appropriately, it can lead to major complications of increased blood loss and poor wound healing. In these two cases, the vulval oedema was found to quickly subside after caesarean delivery. Abdominal delivery is the preferred mode of delivery at Bugando Medical Centre for massive vulval oedema.

\section{Consent}

Consent was obtained from Bugando Ethical committee for publication of these two cases.

\section{Competing interests}

The authors declare that they have no competing interests.

\section{Acknowledgements}

The authors would like to thank Dr. Bani Maheshwari of the Department of Obstetrics and Gynaecology at Weill Cornell Medical College, New York for her assistance.

\section{References}

Bracero, L.A. \& Didomenico, A. (1991) Massive vulvar edema complicating preeclampsia: a management dilemma. Journal of Perinatology 11, 122-125.

Bush, J.P. (1946) Oedema of vulva due to toxaemia of pregnancy. British Medical Journal $2,988$.

Deren, O., Bildirici, I. \& Al, A. (2000) Massive vulvar edema complicating a diabetic pregnancy. European Journal of Obstetrics, Gynecology and Reproductive Biology 93, 209-211.

Geerts, W.H., Pineo, G.F., Heit, J.A., Berggvist, D., Lassen, M.R., Colwell, C.W. \& Ray, J.G. (2004) Prevention of venous thromboembolism: the seventh ACCP Conference on antithrombotic and thrombolytic therapy. Chest 126 (3 Suppl), 338S-400S

Jakobi, P., Friedman, M., Goldstein, I., Zaidise, I. \& Itskovitz-Eldor, J. (1995) Massive vulvar edema in pregnancy. A case report. Journal of Reproductive Medicine 40, 479-481. 
Morris, L.F., Rapini, R.P., Hebert, A.A. \& Katz, A.R. (1990) Massive labial edema in pregnancy. South Med J. 83, 846-848.

Reynolds, D. (2003) Severe gestational edema. Journal of Midwifery Woman's Health 48, 146-148.

Saha, P.K. (2007) Massive vulval edema in pregnancy. Internet Journal of Gynaecology and Obstetrics 6 (2).

Townsend, S.L. (1948) Severe oedema of the vulva in labour. British Medical Journal 1, 344.

Trice, L., Bennert, H. \& Stubblefield, P.G. (1996) Massive vulvar edema complicating tocolysis in a patient with twins. A case report. Journal of Reproductive Medicine 41, 121-124. 